\title{
Plan de diseño organizacional para la eficiencia laboral de la microempresa "EI buen pan" panadería y pastelería
}

\author{
Naslhy Elizabeth Cáceres Falconí \\ ncaceresf@ulvr.edu.ec
}

Yankella Natalie Haro Ponce

yharop@ulvr.edu.ec

Universidad Laica Vicente Rocafuerte de Guayaquil

Guayaquil - Ecuador

\section{RESUMEN}

Desde los inicios de las actividades comerciales de la microempresa "El Buen Pan" Panadería y Pastelería, esta no cuenta con un diseño organizacional por lo que también carece de una cultura organizacional definida y absolutamente todas las actividades se han realizado de manera empírica ya que no se han definido los procesos y funciones internos entre las áreas de la organización. En el presente trabajo de investigación, se propone un Plan de diseño organizacional para la eficiencia laboral de la microempresa “El Buen Pan" Panadería y Pastelería, a través de una investigación descriptiva con enfoque mixto, con la finalidad de definir y establecer de manera formal las funciones y procesos del área de producción y administrativa para que las tareas y responsabilidades dentro de las mismas se realicen eficientemente, logrando un excelente desempeño en la microempresa y un correcto control de los recursos y actividades. De acuerdo a lo mencionado anteriormente, se concluyó que la microempresa “El Buen Pan” Panadería y Pastelería requiere la puesta en marcha de un diseño organizacional para poder alcanzar las metas y objetivos deseados por la misma.

Palabras clave: microempresa; organigrama; manual de funciones; manual de procesos; eficiencia. 


\title{
Organizational design plan for the labor efficiency of the microenterprise "EI buen pan" bakery and pastry shop
}

\begin{abstract}
Since the beginning of the commercial activities of the "El Buen Pan" Bakery and Pastry microenterprise, it does not have an organizational design, so it also lacks a defined organizational culture and all the activities have been carried out empirically since they do not have internal processes and functions have been defined between the areas of the organization. In the present research work, an organizational design plan is proposed for the labor efficiency of the microenterprise "El Buen Pan" Bakery and Pastry Shop, through a descriptive investigation with a mixed approach, with the purpose to define and establish formally the functions and processes of the production and administrative area so that the tasks and responsibilities within them are carried out efficiently, achieving excellent performance in the microenterprise and proper control of resources and activities. According to the aforementioned, it was concluded that the microenterprise "El Buen Pan" Bakery and Pastry requires the implementation of an organizational design in order to achieve the goals and objectives desired by it.
\end{abstract}

Keywords: microenterprise; organization chart; functions manual; procedures manual; efficiency.

Artículo recibido: 30 noviembre. 2021 Aceptado para publicación: 29 diciembre 2021 Correspondencia: ncaceresf@ulvr.edu.ec Conflictos de Interés: Ninguna que declarar 


\section{INTRODUCCIÓN}

La microempresa "EL BUEN PAN" Panadería \& Pastelería dedicada a la producción y comercialización de panes y productos de pastelería, empezó sus actividades comerciales en el año 2013 con solamente un local, el cual se conformaba con 7 trabajadores. Hoy en día, cuenta con una matriz y tres sucursales; las cuales están ubicadas en la ciudad de Guayaquil de la siguiente manera: la matriz, en la Cdla. Martha de Roldós mz. 607 villa 32. La primera sucursal, en la Coop. Madrigal mz. C115 villa 11; la segunda sucursal, en Mapasingue oeste, calle 8va entre la 2da y 3era; y la tercera en la Av. Modesto Luque Rivadeneira (Paraíso de la Flor) Bloque 3 Mz 226 Villa 9. A lo largo de los años, la microempresa ha ido incrementando sus empleados, haciendo un total de 40 colaboradores entre todos los locales.

Sin embargo, la microempresa carece de procesos administrativos y de control ya que no se han definido funciones y procesos internos entre las áreas sino que toda la planificación, organización, dirección y control se ha venido manejando de una manera empírica lo que para algunas áreas, como la de ventas y servicio al cliente, si ha generado buenos resultados; en cambio, tanto para el área administrativa y de producción está ocasionando ciertos inconvenientes como la falta de comunicación entre las dos áreas, ya que el área administrativa no informa con anticipación ni por escrito los días de descanso entre panificadores y horneros, puesto que no mantiene un cronograma de actividades formalmente establecido, provocando que el personal de producción intercambie sus funciones debido a que al no ser comunicados los descansos, existen días en que solo se encuentran en el área de producción los panificadores, y de este grupo uno debe asumir el rol de hornero ya que este se encuentra en descanso, y viceversa, es decir, el hornero asume parte de la producción del panificador que se encuentra en día libre; por lo que existe un intercambio de funciones ya que una persona, ya sea panificador u hornero, debe cumplir con dos roles a la vez para cumplir con la cantidad de producción establecida al día. Cabe recalcar, que cada uno de los productos necesita tiempos exactos para su elaboración, es por esto, que una persona debe cubrir las tareas y funciones del personal que haga falta. Esto ocasiona que el personal culmine sus actividades unos minutos después de la hora de salida establecida mientras que cuando el personal está completo, esto no sucede. 
También se ha notado que la carencia de procesos, procedimientos y políticas está afectando el control del inventario de materia prima, debido a que no existe un registro formal del ingreso y salida del mismo, causando que al momento de que los panificadores empiecen con la elaboración de un producto, estos notan que no cuentan con algún elemento de la materia prima por lo que, esta debe ser abastecida por una de las sucursales más cercanas; sin embargo, esto genera un ligero retraso en el proceso de producción ya que se debe realizar el traslado de materia prima de una sucursal a otra; por otro lado, cuando la materia prima no se encuentra disponible en alguna de las sucursales más cercanas, se debe recurrir a la sucursal que se encuentra más lejos, ocasionando que el traslado se demore aún más y por ende la producción. A pesar de ello, las ventas no se ven afectadas ya que la producción que se realiza en la mañana es para las ventas de la tarde; y por consiguiente, la producción de la tarde para la mañana del siguiente día. A continuación, en la figura 1, se presenta el proceso de producción normal y cuando ocurre retraso:

Figura 1 Proceso de producción con y sin retraso

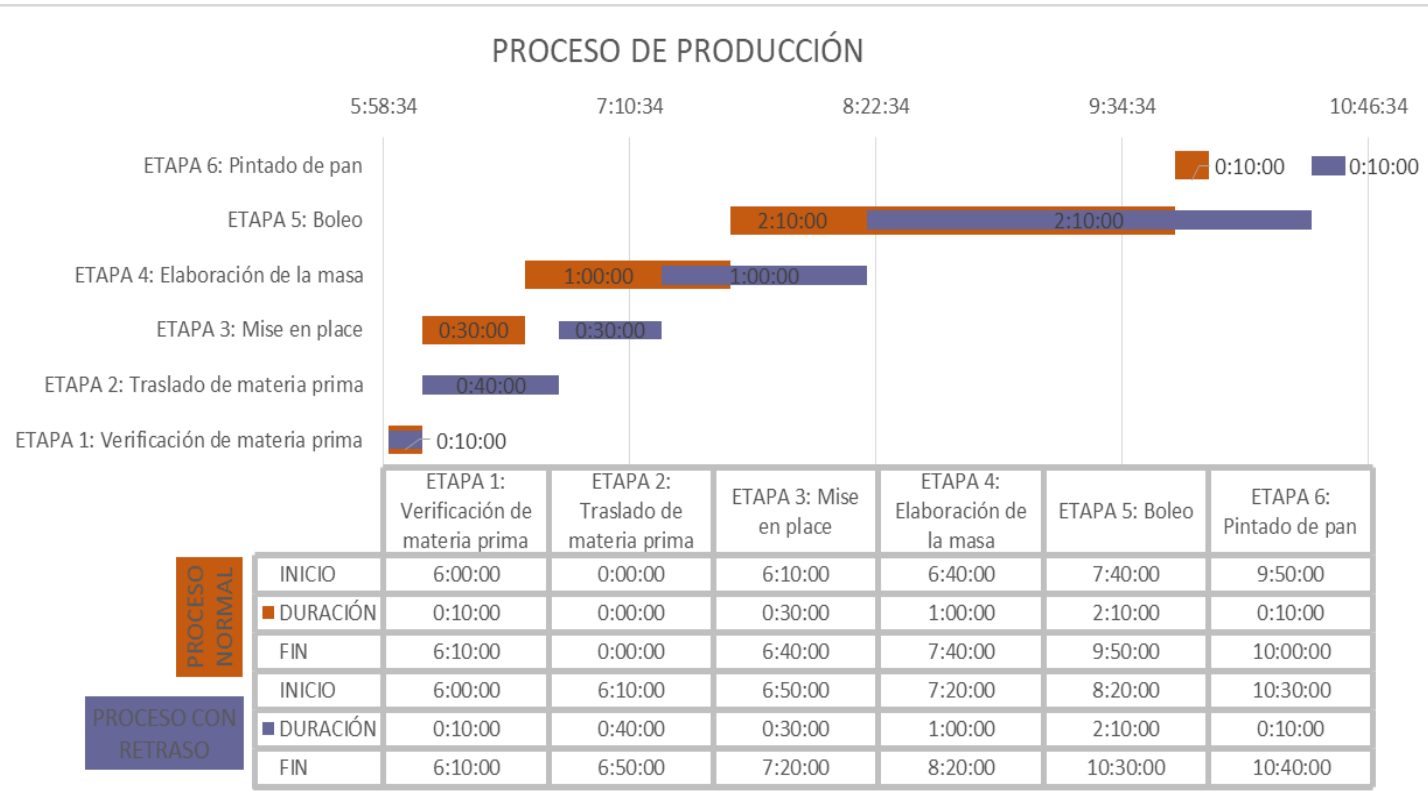

Fuente: “EL BUEN PAN" Panadería \& Pastelería

Analizando la problemática, se determina que es necesario definir y establecer formalmente los procesos y funciones del área administrativa y de producción para que así las tareas y responsabilidades dentro de las mismas se lleven a cabo eficaz y eficientemente, generando un buen desempeño en la microempresa. 


\section{Formulación del Problema}

- ¿Cómo elaborar un plan de diseño organizacional para la eficiencia laboral de la microempresa "EL BUEN PAN" Panadería y Pastelería?

\section{Sistematización del Problema}

- ¿Cómo analizar el estado actual de las áreas de la microempresa "El Buen Pan”?

- ¿De qué manera se pueden diseñar procedimientos y manual de funciones para las diferentes áreas de la microempresa para que exista un adecuado control?

- ¿Cuál será el costo-beneficio de aplicar un diseño organizacional en la microempresa "El Buen Pan"?

\section{Objetivo General}

- Elaborar un plan de diseño organizacional para la eficiencia laboral de la microempresa "EL BUEN PAN" Panadería y Pastelería.

\section{Objetivos Específicos}

- Analizar el estado actual de las áreas de la microempresa "El Buen Pan".

- Diseñar procedimientos y manual de funciones para las diferentes áreas de la microempresa para que exista un adecuado control.

- Determinar el costo-beneficio de aplicar un diseño organizacional en la microempresa "El Buen Pan"

\section{Justificación de la Investigación}

Mejorar la eficiencia de las funciones y tareas de los empleados mediante la aplicación de procesos y procedimientos que establezcan un adecuado control dentro de las áreas con problemas. Logrando así, que la microempresa "EL BUEN PAN" lleve al máximo sus objetivos y continúe ofreciendo productos de calidad ya que actualmente desarrolla sus actividades en base a los estatutos verbales que los propietarios impusieron en su creación, es decir, en el año 2013.

La elaboración de un plan de diseño organizacional para la microempresa será de gran utilidad para que el personal conozca específicamente cuáles son sus funciones y actividades, y de esta manera exista la correcta comunicación entre áreas, la misma que servirá como apoyo para una adecuada gestión y control de los procesos y funciones.

Por otro lado, el diseño de un manual de funciones ayudará a que los procesos dentro del área administrativa y de producción se desarrollen de una manera más eficiente y eficaz, y no se genere intercambio de tareas y responsabilidades entre el personal. 
Es necesario que las áreas estén intercomunicadas, con información de primera mano, por lo que, el diseño y desarrollo de un diagrama de flujo de procesos servirá como herramienta fundamental para que el proceso de adquisición de materia prima se efectúe de una forma más controlada y al momento de producir siempre se cuente con material para producción y no exista ningún tipo de retrasos en el proceso, porque a pesar de contar con productos de excelente calidad y una buena atención al cliente, es importante que la producción sea aún más eficiente ya que si de forma empírica la microempresa ha obtenido buenos resultados en las ventas, estableciendo una correcta planificación y un adecuado control, las ventas podrían incrementar.

El presente proyecto de investigación propone examinar las deficiencias del área administrativa y de producción para que de esta manera se pueda elaborar un diseño organizacional de dichas áreas. Además, mediante técnicas de investigación aplicadas se podrá demostrar que la microempresa carece de un adecuado control dentro de las mismas.

Hoy en día, la relación entre empresas es más competitiva por lo que con el resultado de este proyecto de investigación se llegará a la solución del problema que se ha planteado, el cual es elaborar un plan de diseño organizacional para la eficiencia laboral de la microempresa "EL BUEN PAN" Panadería y Pastelería, trayendo consigo mayor eficiencia en los procesos y funciones para el beneficio de la microempresa.

\section{Idea a Defender}

Al elaborar un plan de diseño organizacional se proporcionará una mejora en la eficiencia laboral de la microempresa "EL BUEN PAN".

\section{Antecedentes}

Según las autoras Centeno y Sánchez, (2021) provee gran aporte al presente proyecto de investigación, el proyecto titulado "Diseño de una estructura organizacional para potenciar la eficiencia y competitividad de la microempresa A DONDE DANILO”. Cuyo objetivo general consistió en: "Determinar la influencia de una estructura organizacional para potenciar la eficiencia y competitividad de la microempresa A DONDE DANILO”. Como conclusión, se obtiene que debido a la informalidad interna en la microempresa, esta genera deficiencia en sus trabajadores, ya que no proporcionan un buen desempeño, viéndose reflejado en la poca responsabilidad que tienen para realizar las tareas; 
asimismo, se crea una incompatibilidad entre trabajadores y se llega al ausentismo puesto que no existe una identidad organizacional bien definida.

De acuerdo con los autores Canteral y Moran, (2019) en su proyecto "Plan de diseño organizacional para la empresa Hirometalsa S.A.”. Determinaron que el objetivo general debía basarse en "Elaborar un plan de diseño organizacional para la empresa Hirometalsa S.A.”. En fin, se deduce que la falta de control en el inventario ocasiona que no se estime de manera correcta el stock de la materia prima provocando que las órdenes de producción no se cumplan a cabalidad y esto genere pérdidas proporcionales en la microempresa.

Conforme la autora Madrid, (2017) en su proyecto "Implementación de la estructura organizacional y manuales funcionales de una empresa ubicada en el norte de Quito caso: Rincón del Gaucho", su objetivo general fue "Llevar un control de calidad semestral de los productos y servicios del personal generando un indicador de calidad del servicio el cual será medido cada 6 meses y en el que se deberá superar el 60\% de excelencia para garantizar el producto gastronómico que ofrecemos así como la grata experiencia que se genera al visitar nuestros locales". Tanto este trabajo como el proyecto presente de investigación, es considerable establecer una estructura organizacional para poder determinar los papeles que tomaran los diferentes miembros de las entidades para que logren trabajar en equipo de manera óptima y sean capaces de alcanzar las metas fijadas. La autora Salcedo, (2016) presenta su proyecto "Propuesta de Estructura Organizacional para la Empresa Camaronera Jemaredli S.A.", con el objetivo general "Diseñar una propuesta de modelo organizacional adecuado para la empresa camaronera Jemaredli S.A, que permita el mejorar el desarrollo empresarial". La empresa presenta problemas en el área administrativa porque no tienen una estructura organizacional constituida en el que puedan estar detallados los procedimientos y funciones para que los colaboradores puedan realizar las actividades de manera competente.

El autor Ormaza, (2016) propone su proyecto "Propuesta de un diseño organizacional para la hacienda Santa Mónica ubicada en el Recinto Oro Verde - Cantón Quinsaloma”. Comprende como objetivo general "Elaborar una propuesta de diseño organizacional para la Hacienda Agrícola Santa Mónica dedicada a la producción de cacao”. La problemática radica en que no existe una estructura organizacional definida y la persona encargada de dirigir la hacienda no cuenta con recursos y conocimientos administrativos lo que 
ocasiona que los colaboradores no tengan a alguien que los dirija oportunamente y por esto no realizan las actividades de manera apropiada.

\section{Teorías}

- Jones (2008) define diseño organizacional como:

"El diseño organizacional es el proceso por medio del cual los gerentes seleccionan y administran los aspectos de estructura y cultura para que una organización pueda controlar las actividades necesarias con las que logra sus metas". (pág. 9)

\section{- Según Saroka \& Ferrari (2015) un organigrama es:}

"Es la representación parcial, mediante un diagrama, de la estructura formal de una organización, en él se muestran las funciones, sectores, jerarquías y dependencias internas”. (pág. 5)

- Jones (2008) argumenta que la cultura organizacional es:

"El conjunto de valores y normas compartidos que controla las interacciones entre los integrantes de la organización y con los proveedores, clientes y otras personas externas a la misma" (pág. 8)

- Franklin (1998) expresa que el manual de funciones es:

"Un documento formal que agrupa las diversas descripciones de los puestos de trabajo de una organización. Es el resultado de un estudio de los puestos de trabajo de una organización para llevar a cabo una adecuada gestión de las personas”. (pág. 88)

\section{- Para Mallar (2010) un proceso}

"es un conjunto de actividades de trabajo interrelacionadas, que se caracterizan por requerir ciertos insumos (inputs: productos o servicios obtenidos de otros proveedores) y actividades específicas que implican agregar valor, para obtener ciertos resultados (outputs)". (pág. 5)

\section{- Para Franklin (2009) los manuales de procedimientos}

"constituyen un documento técnico que incluye información sobre la sucesión cronológica y secuencial de operaciones concatenadas entre sí, que se constituyen en una unidad para la realización de una función, actividad o tarea específicas en una organización”. (pág. 245) 
- George Terry define a los diagramas de procedimientos, también conocidos como flujogramas, como "la representación gráfica que muestra la sucesión de los pasos de que consta un procedimiento". (Como se citó en Luna, 2014 (pág. 90)).

\section{MATERIALES Y MÉTODOS}

\section{Tipo de Investigación}

Se pretende utilizar la investigación descriptiva para recabar información a través de las diferentes técnicas de investigación para determinar qué tan importante es desarrollar un plan de diseño organizacional, puesto que en base a esto se podrán encontrar soluciones que beneficien a la microempresa, diseñar procesos y procedimientos, aplicar políticas, estableces objetivos para así incrementar su eficiencia. Según Hernández Sampieri (2018), la investigación descriptiva busca detallar de manera específica aquellas propiedades, características, perfiles y/o comportamientos que pueden tener las personas, grupos o procesos que se encuentren sujetos a un análisis. (pág. 92)

\section{Enfoque de la Investigación}

Para el presente proyecto de investigación, se utilizó un enfoque mixto; puesto que la aplicación de un enfoque tanto cualitativo como cuantitativo fue necesario para recabar información que ayude al desarrollo del mismo. Un enfoque cualitativo se basa en obtener información o datos pero sin enfocarse mucho en su cuantificación, puesto que observa, analiza y describe los fenómenos o situaciones que se presentan y son sujetas a estudio. (Ñaupas et al., 2018); el enfoque cuantitativo se encarga de recolectar información y datos que serán medidos numérica y estadísticamente, ya que serán usadas para la verificación de hipótesis y/o teorías. (Hernández Sampieri, 2014)

\section{Técnicas de Investigación}

- Para el presente proyecto de investigación, se realizó una encuesta a los colaboradores del área de producción de la microempresa "El Buen Pan" para comprender lo que sucede en esa área, conocer sus opiniones y obtener información importante referente a la forma en la que se llevan a cabo las funciones y los procesos dentro de la microempresa.

- Se utilizó la entrevista para recabar información relevante con los administradores de la microempresa "El Buen Pan", ya que conocen todos los aspectos positivos y negativos de la organización. 
- Se llevó a cabo la observación directa en las instalaciones de la microempresa "El Buen Pan", a través de este método se pudo evidenciar cómo es el funcionamiento de la microempresa, la comunicación organizacional, constatar lo que sucede en el área de producción y conocer la situación actual de la microempresa.

\section{Instrumentos de Investigación}

- Por un lado, se realizó un cuestionario para aplicar una encuesta al personal de la microempresa "El Buen Pan", la cual está diseñada por 8 preguntas elaboradas con anterioridad, las mismas que son de carácter cerradas. Por otro lado, las preguntas elaboradas para las entrevistas a los administradores y jefes de producción son de carácter abiertas, ya que de esta forma se obtiene información más específica y detallada. Mediante las encuestas se puede obtener información estadística sobre la situación que está afectando y con la entrevista se puede conocer a profundidad el problema.

- Se utilizó la ficha de observación durante el estudio para poder recabar toda la información necesaria, conocer las características y todo lo que influye dentro de la microempresa "El Buen Pan", de manera especial en el área de producción que es donde se están generando los inconvenientes.

\section{Población}

Considerando que el número de individuos se conocen, es decir, son identificables, se determina a la población como finita. Alrededor de 34 trabajadores, entre todos los locales, estuvieron sujetos al desarrollo de las encuestas; mientras que las entrevistas fueron aplicadas para los dos administradores y cuatro jefes de producción.

\section{Método de Análisis de Datos}

Para efectos de un correcto análisis de los datos, se empleó el software Microsoft Excel, en donde se tabuló y agrupó la información mediante tablas y gráficos los cuales muestran los datos obtenidos en la investigación. De forma que, se presenta de manera escrita la información relevante de cada dado encontrado la cual también es tabulada, es decir, se presenta en manera de porcentaje dicha información.

\section{RESULTADOS Y DISCUSIÓN}

El presente análisis reúne información acerca del diagnóstico de las herramientas de investigación utilizadas, como la encuesta, entrevista y fichas de observación. 
- Los administradores comentaron que no han estructurado formalmente la microempresa porque no tienen los conocimientos necesarios ya que cuando empezaron con el primer local, lo hicieron de manera empírica y así lo desarrollan hasta la actualidad. Sin embargo, desde que les comunicamos lo importante que es tener un diseño organizacional, ellos consideran que les gustaría establecerlo porque sería de gran ayuda para mejorar algunos aspectos y sobre todo que puedan administrar de una mejor manera la microempresa.

- La comunicación entre los administradores y trabajadores es efectiva, sin embargo, cuando los administradores planifican los días libres de los trabajadores muchas veces no lo notifican con anticipación y esto ocasiona que los trabajadores intercambien las funciones para poder sacar la producción a tiempo.

- Los trabajadores no tienen un manual de funciones para realizar sus actividades diarias, sino que lo hacen de manera empírica o porque los administradores les han explicado verbalmente.

- La mayoría de los trabajadores consideran que un manual de funciones podría ser de gran apoyo para los trabajadores nuevos e incluso para ellos mismos para que puedan realizar sus actividades diarias de mejor manera.

- Los trabajadores a pesar de que tienen una buena relación con la dirección administrativa de la empresa, consideran que si existen aspectos que deberían mejorar, en cuanto a la planificación y organización de los días libres y el control de inventario.

- De acuerdo a los trabajadores que fueron encuestados y entrevistados, expresaron que es una gran idea desarrollar un manual de funciones y procesos para tener una base de lo que deben desarrollar en su puesto de trabajo. También es esencial que, al diseñar un manual de funciones y procesos, se realicen capacitaciones para que los trabajadores antiguos y nuevos puedas conocer de forma clara, cada una de las funciones relacionadas con el puesto de trabajo para el que fueron contratados.

\section{CONSIDERACIONES FINALES}

\section{Conclusión}

- Dando respuesta al Objetivo \#1: “Analizar el estado actual de las áreas de la microempresa El Buen Pan”, se puede concluir que pese a que es una microempresa que lleva algunos años en el mercado, esta no se encuentra formalmente organizada 
ya que en todo este tiempo, por falta de información y conocimientos, no se han tomado las medidas necesarias para mejorar el funcionamiento interno mediante un diseño organizacional definido, generando inconvenientes los cuales deben ser solucionados de manera inmediata.

- En base al Objetivo "2: "Diseñar procedimientos y manual de funciones para las diferentes áreas de la microempresa para que exista un adecuado control", se concluye que es indispensable desarrollar y poner en marcha un diseño organizacional para que las actividades, funciones o tareas sean controladas puesto que la falta de esto y por ende de comunicación, ha ocasionado que algunos trabajadores intercambien en varias ocasiones sus funciones, sumándole a ello el desconocimiento de los trabajadores de las tareas específicas a realizar en su puesto de trabajo, ya que la microempresa al no poseer manuales de funciones y de procesos formales genera retrasos en la producción; por lo que, diseñar estos manuales enfocará la eficiencia en los procesos y los colaboradores tendrán conocimiento de sus tareas específicas.

- Para dar respuesta al Objetivo \#3: "Determinar el costo-beneficio de aplicar un diseño organizacional en la microempresa El Buen Pan", se puede concluir que cada costo al que se va a incurrir para la mejora de la situación cumple con las expectativas de los beneficios a obtener, teniendo un costo total de la propuesta de \$906.20, el cual será empleado en el segundo semestre del año 2022. La microempresa a pesar de no contar con un diseño organizacional, posee una cartera de clientes que se ha mantenido fiel durante todos estos años, es por eso que gracias a las buenas ventas y mediante la optimización de recursos, genera una utilidad neta de aproximadamente el 30\%, por lo que se considera que la inversión en un diseño organizacional no afectará económicamente a "El Buen Pan”.

\section{Recomendaciones}

- Para mejorar varios aspectos de la organización, se determinó que es preciso definir y establecer formalmente las funciones y procesos del área de producción y administrativa para que las tareas realizadas por los colaboradores se desarrollen de manera eficiente, logrando un buen desempeño entre áreas.

- Es esencial el desarrollo de un diseño organizacional ya que esto posibilita que las tareas se lleven a cabo de una mejor manera para alcanzar los resultados que la microempresa desea. Así se puede determinar una correcta estructura organizacional. 
- Establecer un organigrama, manual de funciones y procedimientos, de esta manera se podrá organizar el trabajo eficientemente, también ayuda en el proceso de comunicación, desarrollo e integración entre áreas.

- También es indispensable que tanto administradores como los colaboradores de la microempresa realicen las capacitaciones nombradas en el capítulo IV de esta investigación ya que es primordial que puedan adquirir conocimientos, habilidades y herramientas para realizar sus actividades eficientemente.

- Una vez analizados todos los factores por mejorar, es necesario que se implemente el Plan de diseño organizacional para la microempresa. Con esta investigación y las herramientas ya mencionadas, la organización estará preparada en alcanzar un nivel mayor en sus actividades comerciales y el crecimiento de la misma.

- Es fundamental que, al realizar algún cambio para mejorar algún factor de la microempresa, se lleve un adecuado seguimiento para verificar que las acciones que se tomaron son las correctas y necesarias para el bienestar de la organización.

\section{PROPUESTA}

\section{Presentación del Diseño organizacional}

\section{Misión}

Somos una microempresa dedicada a la producción y comercialización de productos de panadería y pastelería artesanal, comprometidos con nuestros clientes para garantizar la satisfacción mediante la calidad y buen sabor.

\section{Visión}

Ser una empresa reconocida a nivel nacional en el mercado de panadería y pastelería artesanal cumpliendo con las expectativas de nuestros clientes y garantizar su satisfacción mediante la calidad de nuestros productos.

\section{Organigrama}

En la figura 2 se muestra el organigrama general vertical que se propone para que la microempresa conozca los niveles jerárquicos y a su vez se mejore la comunicación entre las áreas, ya que se presentan de manera ordenada; el mismo que se aplicará para matriz y sucursales. 
Figura 2 Organigrama General

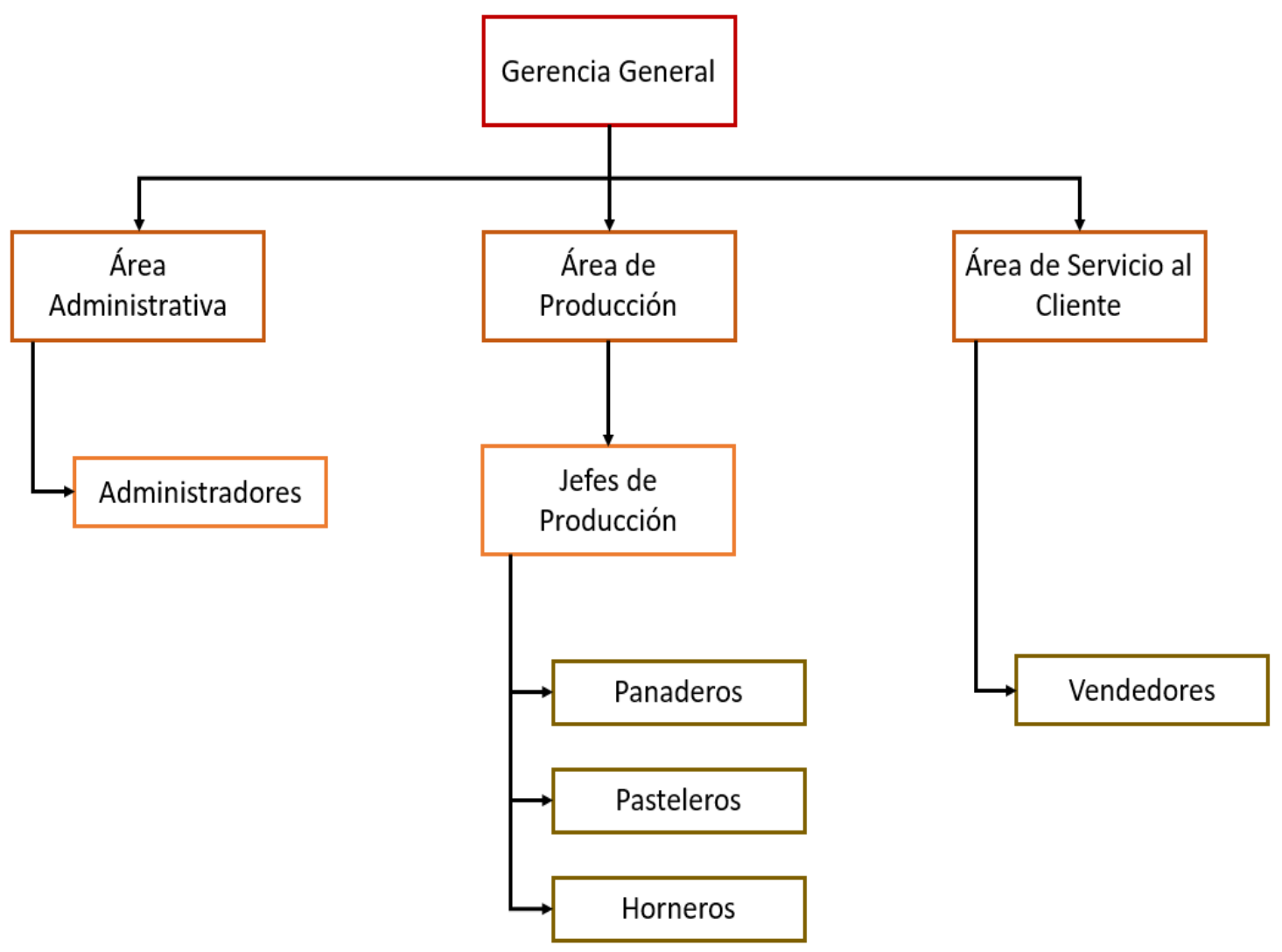

Elaborado por: Haro y Cáceres, 2022

\section{Manual de funciones}

Se propone un manual de funciones aplicable a la microempresa "El Buen Pan", ya que de esta maneja se mejorará la comunicación entre el personal y existirá un orden en la designación de los puestos de trabajo, debido a que cada colaborador tendrá conocimiento sobre las funciones, actividades y responsabilidades que posee al momento de ejercer el cargo; de esta manera, no habrá intercambio de funciones puesto que cada uno sabe formalmente lo que debe hacer. La Tabla 1 muestra un ejemplo: 
Tabla 1 Manual de funciones

\begin{tabular}{|c|c|c|}
\hline $\begin{array}{c}\text { "El Buen Pan" } \\
\text { Panadería y } \\
\text { Pastelería }\end{array}$ & $\begin{array}{l}\text { MANUAL DE } \\
\text { FUNCIONES }\end{array}$ & $\begin{array}{c}\text { Fecha: } 3 / 12 / 2021 \\
\text { Versión } 1.0\end{array}$ \\
\hline \multicolumn{3}{|c|}{ DESCRIPCIÓN DEL CARGO } \\
\hline \multicolumn{3}{|c|}{ Cargo: Jefe de Producción (Maestro panificador) } \\
\hline \multirow{2}{*}{\multicolumn{3}{|c|}{$\begin{array}{l}\text { Detalle: Supervisar y elaborar productos afines a la panadería, aplicando diversas técnicas } \\
\text { Tino de cargo: Operativo }\end{array}$}} \\
\hline \multicolumn{2}{|c|}{ Tipo de cargo: Operativo } & \\
\hline \multicolumn{3}{|c|}{$\begin{array}{l}\text { - Mreparación de las masas para los diferentes tipos de pan } \\
\text { - Mantener la limpieza, cuidado y mantenimiento de los utensilios de trabajo } \\
\text { - Amasado y elaboración de panes de especialidad }\end{array}$} \\
\hline \multicolumn{3}{|c|}{ PERFIL DE CARGO } \\
\hline Edad: 20 a 35 años & \multicolumn{2}{|c|}{$\begin{array}{l}\text { Formación Académica: Bachiller y Estudios de } \\
\text { Panificación Profesional }\end{array}$} \\
\hline Género: Masculino & \multicolumn{2}{|c|}{ Experiencia laboral: Mínimo 2 años en cargos similares } \\
\hline \multicolumn{3}{|c|}{ Conocimientos adicionales: Técnicas europeas de panificación } \\
\hline Firma de responsabilidad: Sí & Manejo de dinero: No & Manejo de máquinas: Sí \\
\hline Reporta a: Administradores & $\begin{array}{l}\text { Supervisa a: panaderos y } \\
\text { horneros a su cargo }\end{array}$ & $\begin{array}{l}\text { Supervisado por: } \\
\text { Administradores }\end{array}$ \\
\hline Elaborado por: & Revisado por: & Aprobado por: \\
\hline
\end{tabular}

Elaborado por: Haro y Cáceres, 2022

\section{Manual de procesos}

A continuación en la figura 3 se muestran los procesos que deben realizarse para la actividad principal de cada área. Se refleja el proceso que se realiza para la adquisición de la materia prima. Adicionalmente, se muestra cada paso que se realiza al momento de la producción, y también se detalla el proceso que se desarrolla para la venta de un producto. 
Figura 3 Manual de procesos

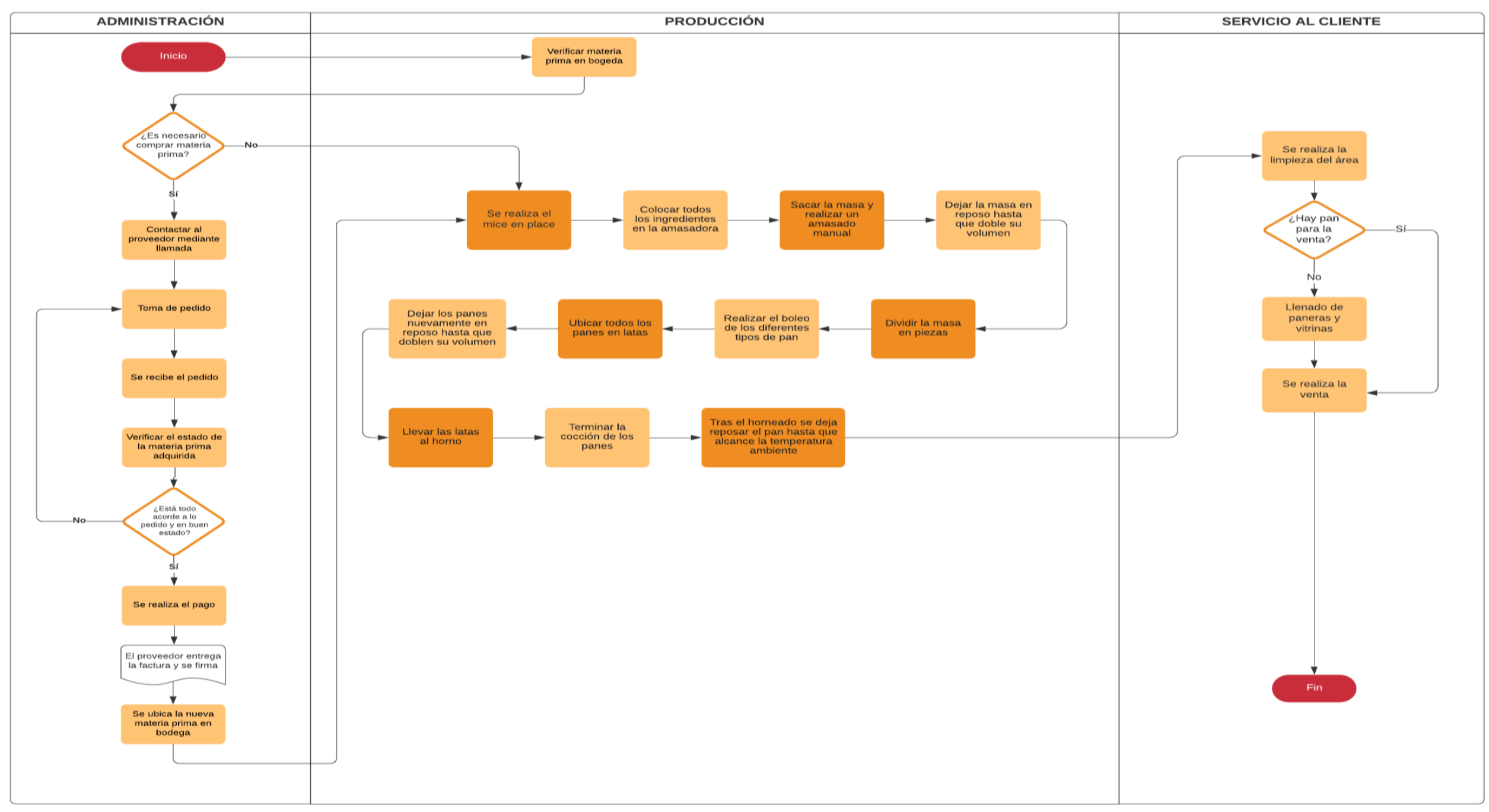

Elaborado por: Haro y Cáceres, 2022

Ciencia Latina Revista Científica Multidisciplinar, Ciudad de México, México.

ISN 2707-2207 / ISSN 2707-2215 (en línea), enero- febrero, 2022, Volumen 6, Número 1.

https://doi.org/10.37811/cl_rcm.v6i1.1555 p986 


\section{Fichas de evaluación}

Las tablas 2 y 3 muestran las fichas de evaluación propuestas para controlar y evaluar los cambios que se implementen en la microempresa mediante el diseño organizacional. De esta manera, se estará evaluando cómo el personal se ha ido acoplando y cómo ha ayudado en la comunicación entre trabajadores. Del mismo modo, los procesos estarán siendo evaluados periódicamente para determinar si estos son adecuados y si existe una mejora con su aplicación; generando mayor eficiencia en las actividades.

Tabla 2 Ficha de evaluación a trabajadores

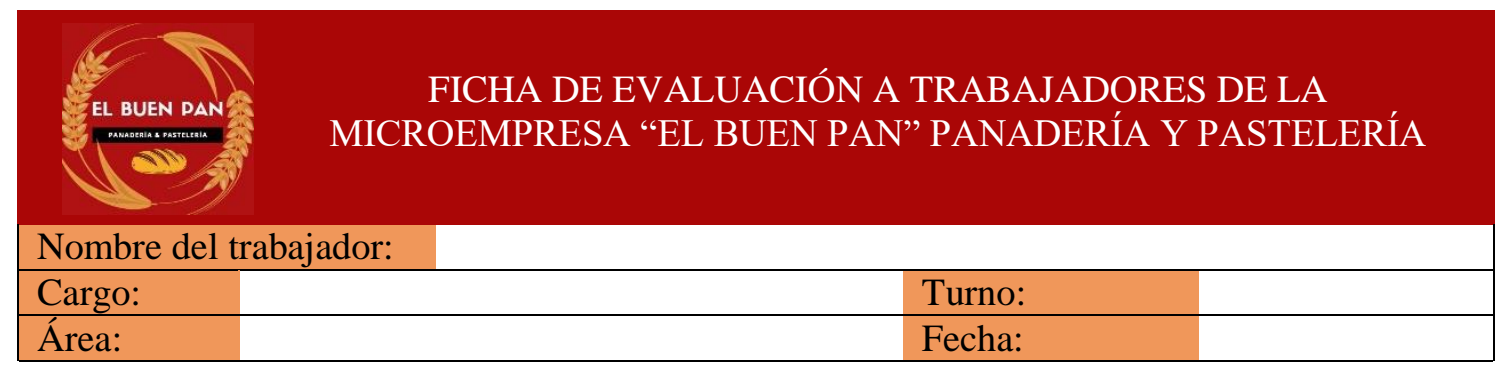

\begin{tabular}{|c|c|c|c|c|}
\hline \multicolumn{5}{|c|}{ ESCALA DE VALORACIÓN } \\
\hline Siempre & Casi siempre & Muchas veces & Algunas veces & Nunca \\
\hline 5 & 4 & 3 & 2 & 1 \\
\hline
\end{tabular}

\begin{tabular}{|c|l|c|c|c|c|c|}
\hline \multicolumn{2}{|c|}{ DESEMPEÑO DEL TRABAJADOR } & \multicolumn{3}{c|}{ PUNTUAĆN } \\
\hline 1 & Tiene conocimiento de sus funciones en el puesto de trabajo & & & & & \\
\hline 2 & Demuestra dominio en sus actividades & & & & \\
\hline 3 & Manifiesta buena coordinación con su equipo de trabajo & & & & \\
\hline 4 & Cuenta con una adecuada comunicación con sus superiores & & & & & \\
\hline 5 & Cuenta con una adecuada comunicación con sus compañeros & & & & \\
\hline 6 & $\begin{array}{l}\text { Realiza sus actividades en base a los diagramas de procesos } \\
\text { establecidos }\end{array}$ & & & & \\
\hline 7 & Es eficiente y eficaz en sus actividades diarias & & & & \\
\hline 8 & $\begin{array}{l}\text { Demuestra puntualidad con el horario de trabajo que se le ha } \\
\text { establecido }\end{array}$ & & & & \\
\hline TOTAL DE PUNTOS & & & & \\
\hline Evaluado por: & & & & \\
\hline
\end{tabular}

Elaborado por: Haro y Cáceres, 2022 
Tabla 3 Ficha de evaluación de los procesos

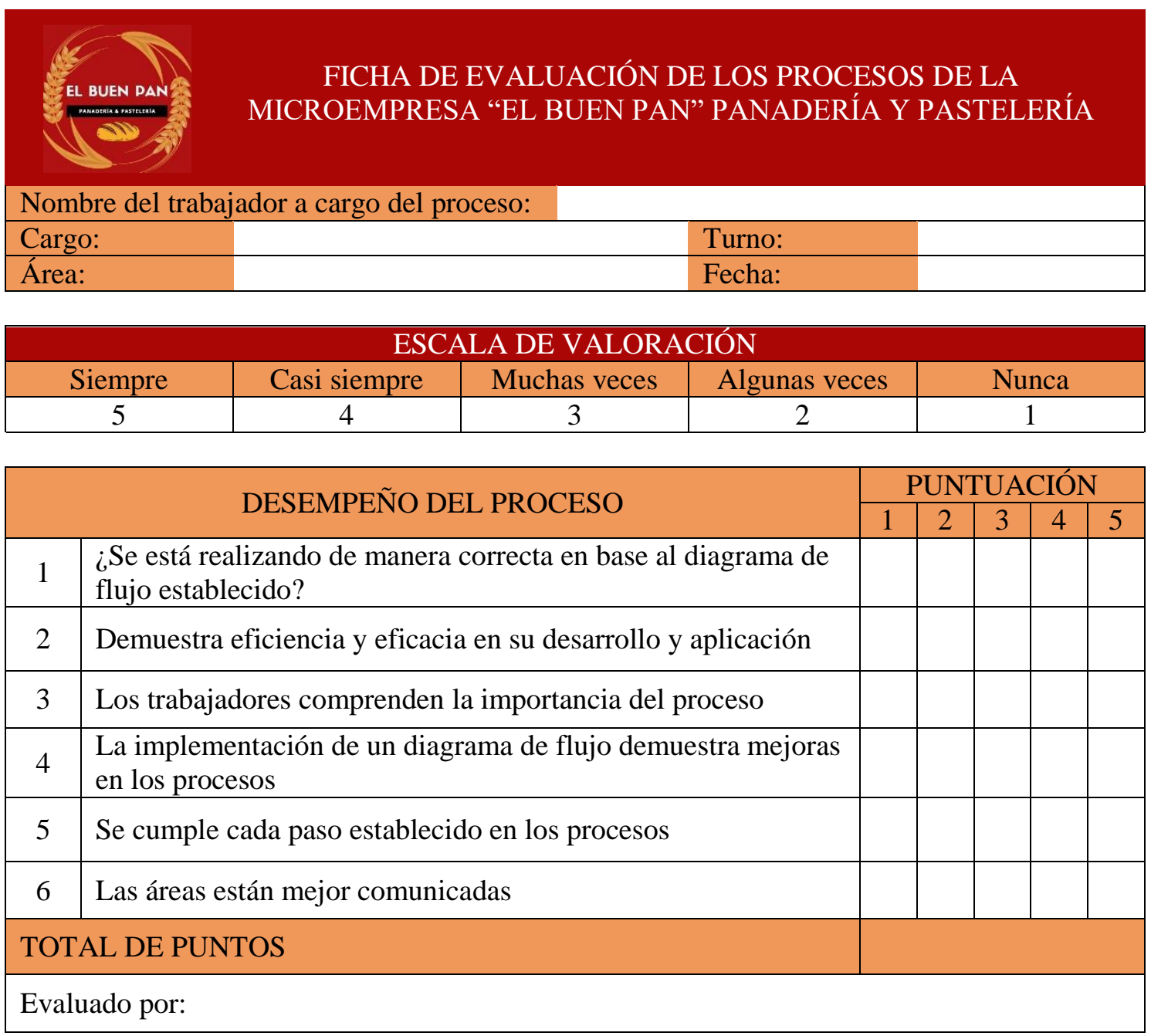

Elaborado por: Haro y Cáceres, 2022

La ficha de evaluación a los trabajadores consta de ocho aspectos para ser controlados y evaluados, teniendo una puntuación máxima de 40 puntos y el puntaje mínimo a ser considerado es de 24. Asimismo, la ficha de evaluación de los procesos tiene su puntaje máximo y mínimo, siendo 30 y 18 respectivamente.

\section{Capacitaciones}

La microempresa "El Buen Pan" tendrá el compromiso de capacitar a todo su personal, desde el área administrativa hasta la operativa, para que así cada uno de ellos pueda comprender y familiarizarse con los cambios organizacionales que se estarán efectuando. Es por esto, que a continuación la tabla 4 muestra las capacitaciones que se proponen para que se pueda mostrar de manera óptima el diseño organizacional a los trabajadores, así como mejoras en comunicación y procesos. 
Tabla 4 Propuesta de capacitaciones

\begin{tabular}{ccccccccc}
\hline \multicolumn{6}{c}{ PROPUESTA DE CAPACITACIONES AL PERSONAL DE “EL BUEN PAN” } \\
\hline Descripción & Modalidad & Capacitador & Duración & Área & $\begin{array}{c}\mathbf{N}^{\circ} \text { de } \\
\text { personas }\end{array}$ & $\begin{array}{c}\text { Costo } \\
\text { Unitario }\end{array}$ & $\begin{array}{c}\text { Costo } \\
\text { total }\end{array}$ \\
\hline $\begin{array}{c}\text { Fundamentos } \\
\text { para un Diseño } \\
\text { Organizacional }\end{array}$ & Online & Udemy & 50 horas & Administrativa & 2 & $\$ 44$ & $\$ 88$ \\
\hline $\begin{array}{c}\text { Gestión de } \\
\text { Inventarios }\end{array}$ & Online & Netzun & 3 horas & $\begin{array}{c}\text { Administrativa } \\
\text { y Operativa }\end{array}$ & 6 & $\$ 18$ & $\$ 108$ \\
\hline $\begin{array}{c}\text { Coaching para } \\
\text { el liderazgo y } \\
\text { trabajo en } \\
\text { equipo }\end{array}$ & Online & Netzun & 3 horas & Operativa & 38 & $\$ 18$ & $\$ 684$ \\
\hline
\end{tabular}

TOTAL DE LA PROPUESTA

Elaborado por: Haro y Cáceres, 2022

\section{Presupuesto}

Tabla 5 Presupuesto de la propuesta

\begin{tabular}{|c|c|c|c|c|c|c|}
\hline & \multirow{2}{*}{ DETALLE } & \multicolumn{3}{|c|}{ CANTIDAD } & \multirow{2}{*}{ C/. UNITARIO } & \multirow{2}{*}{ TOTAL } \\
\hline & & HORAS & HOJAS & PERSONAS & & \\
\hline \multirow{2}{*}{ JULIO } & \multirow{2}{*}{\begin{tabular}{|l|} 
Impresión del diseño organizacional \\
Curso Fundamentos para un Diseño Organizacional
\end{tabular}} & & 12 & 40 & 0.05 & 24.00 \\
\hline & & 50 & & 2 & 44.00 & 88.00 \\
\hline AGOSTO & Capacitación online Netzun: Gestión de Inventarios & 3 & & 6 & 18.00 & $\$ \quad 108.00$ \\
\hline SEPTIEMBRE & $\begin{array}{l}\text { Capacitación online Netzun: Coaching para el } \\
\text { liderazgo y trabajo en equipo }\end{array}$ & 3 & & 38 & 18.00 & $\$ \quad 684.00$ \\
\hline NOVIEMBRE & Impresión de fichas de evaluación & & 44 & & 0.05 & 2.20 \\
\hline & TOTAL & & & & & $\$ \quad 906.20$ \\
\hline
\end{tabular}

Elaborado por: Haro y Cáceres, 2022 


\section{Costo/Beneficio}

Tabla 6 Costo/Beneficio

\section{Costo}

$\$ 24.00$ por la impresión del diseño organizacional para todos los trabajadores

$\$ 88.00$ para capacitar de manera online a los dos administradores en un curso de Fundamentos para un diseño organizacional

$\$ 108.00$ para capacitación a trabajadores, específicamente a administradores y jefes de producción

$\$ 684.00$ para capacitar a trabajadores

\$2.20 para la impresión de las fichas de evaluación

\section{Beneficio}

Todo el personal tendrá conocimiento de cuál es la misión, visión, valores; sabrá cuáles son sus funciones específicas de acuerdo al manual de funciones y cómo deben realizarse los procesos en base al manual. Asimismo, conocerá la jerarquía a través del organigrama de la microempresa, facilitando la comunicación entre trabajadores y por ende, áreas.

Es esencial que los administradores aprendan acerca de lo que es un diseño organizacional porque es importante que puedan ponerlo en práctica en "El Buen Pan" ya que ayuda a formalizar los procedimientos dentro de la microempresa, brinda orientación y también permite que los colaboradores conozcan acerca de la organización.

A través de esta capacitación, los administradores y jefes de producción podrán comprender que llevar un correcto manejo de inventarios es muy importante para la productividad de la microempresa. Es indispensable que puedan conocer todos los procesos que son vitales para lograr alcanzar una gestión eficiente en el manejo de inventario, manteniendo un balance correcto de existencias en bodega.

Los trabajadores podrán mejorar la manera en la que han estado trabajando en equipo, mantener un buen liderazgo las veces que se lo requiera. La eficiencia y eficacia incrementará porque el trabajador se sentirá comprometido con su trabajo, ya que existirá armonía y buen clima laboral al momento de realizar las actividades.

Una evaluación general de los cambios efectuados permitirá conocer cuáles han sido los avances y/o las mejoras en dicha aplicación. Dará pie a tomar más decisiones mediante el análisis y control de los resultados, generando estrategias para continuar con una buena organización tanto interna como externa. 
Plan de diseño organizacional...

\section{LISTA DE REFERENCIAS}

Arias Coello, A. (s.f.). La Gestión de los Procesos. Obtenido de https://webs.ucm.es/centros/cont/descargas/documento10142.pdf

Beltrán Jaramillo, J. M. (2009). Indicadores de Gestión. Herramientas para lograr la competitividad. Bogotá: 3R Editores.

Bernal Torres, C. A. (2016). Metodología de la Investigación. Colombia: Pearson.

Bravo Carrasco, J. (2011). Gestión de Procesos. Santiago de Chile: Evolución S.A.

Brume, M. (2019). Estructura organizacional. Barraquilla: Sello Editorial: Institución Universitaria Itsa.

Centeno, G., \& Sánchez, A. (2021). Tesis Pregrado "Diseño de una estructura organizacional para potenciar la eficiencia y competitividad de la microempresa A donde Danilo". Obtenido de Repositorio ULVR: http://repositorio.ulvr.edu.ec/bitstream/44000/4459/1/T-ULVR-3630.pdf

Figueroa Delgado, J. F. (Agosto de 2020). Tesis Posgrado. PLAN DE MARKETING OPERATIVO EN LA DULCERÍA TRADICIONAL LOS ALMENDROS AGENCIA ROCAFUERTE . Obtenido de Repositorio ESPAM: http://repositorio.espam.edu.ec/bitstream/42000/1339/1/TTMADME01D.pdf

Flores, J. M. (10 de julio de 2018). Derecho Ecuador. Recuperado el 2021, de Microempresa: https://www.derechoecuador.com/microempresa

Franklin Fincowsky, E. B. (2009). Organización de Empresas. México: McGraw-Hill Interamericana. Obtenido de https://naghelsy.files.wordpress.com/2016/01/enrique-franklin-organizacion-deempresas.pdf

Franklin, E. (1998). Organizacion de empresas . Mexico: McGRAW-HILL INTERAMERICANA EDITORES, S.A.

García Dihigo, J. (2016). Metodología de la Administración para Administradores. Bogotá: Ediciones de la U. Obtenido de https://books.google.es/books?hl=es\&lr=\&id=JiwaEAAAQBAJ\&oi=fnd\&pg=P $\mathrm{A} 1 \& \mathrm{dq}=$ tecnicas + de + investigacion $\&$ ots $=\mathrm{bln} 0 \mathrm{H} 0 \mathrm{AhxU} \& \mathrm{sig}=\mathrm{c}-$ aCsxHm2QNMHCaZLr1Gxo4gFWQ\#v=onepage\&q\&f=false

García et al. (2019). Indicadores de Eficacia y Eficiencia en la gestión de procura de materiales en empresas del sector construcción del Departamento del Atlántico, 
Colombia. Revista Espacios, 1-11. Obtenido de http://www.revistaespacios.com/a19v40n22/a19v40n22p16.pdf

Grande Esteban, I., \& Abascal Fernández, E. (2017). Fundamentos y Técnicas de Investigación Comercial. Madrid: ESIC Editorial. Obtenido de https://books.google.es/books?hl=es\&lr=\&id=zbaaDgAAQBAJ\&oi=fnd\&pg=P A19\&dq=tecnicas+de+investigacion\&ots=U2QS1NIIy9\&sig=3_B742_H2XQ2NGXr6eLNf-AV0\#v=onepage \&q\&f=false

Gutiérrez Aragón, Ó. (2016). Fundamentos de Administración de Empresas. Barcelona: Ediciones Pirámide. Obtenido de http://104.207.147.154:8080/bitstream/54000/1242/1/Guti\%c3\%a9rrez-

Administraci\%c3\%b3n\%20de\%20empresas.pdf

Henry, H. W. (1980). Appraising a company's strengths and weaknesses.

Hernández Sampieri, R. (2014). Metodología de la Investigación. México: McGrew-Hill Education.

Hernández Sampieri, R. (2018). Metodología de la Investigación. México: McGraw-Hill Education.

ISO 9000, N. I. (15 de Septiembre de 2015). Recuperado el 2021, de http://www.umc.edu.ve/pdf/calidad/normasISO/ISO\%209000-2015.pdf

Iturralde, R. (2017). Administración para Pymes. Guayaquil: Universidad Laica VICENTE ROCAFUERTE de Guayaquil.

Jones, G. (2008). TEORÍA ORGANIZACIONAL. México: PEARSON EDUCACIÓN.

Luna González, A. (2014). Proceso Administrativo. México: Patria. Obtenido de https://books.google.com.gt/books?id=b8_hBAAAQBAJ\&printsec=copyright\& $\mathrm{hl}=\mathrm{es} \# \mathrm{v}=$ onepage $\& \mathrm{q} \& \mathrm{f}=$ false

Maldonado, J. Á. (2018). ISSUU. Recuperado el 2021, de Gestión por Procesos: https://issuu.com/joseangelmaldonado8/docs/gesti_n_de_procesos_2018_

Mallar, M. Á. (2010). LA GESTIÓN POR PROCESOS: UN ENFOQUE DE GESTIÓN EFICIENTE. Revista Científica "Visión de Futuro", 13, 1-23. Obtenido de https://www.redalyc.org/pdf/3579/357935475004.pdf

Marcó, F., Loguzzo, H. A., \& Fedi, J. L. (2016). Introducción a la Gestión y Administración en las Organizaciones. Argentina: Universidad Nacional Arturo Jauretche. Obtenido de https://biblioteca.unaj.edu.ar/wp- 
content/uploads/sites/8/2017/02/Introduccion-gestion-y-administracionorganizaciones.pdf

Ñaupas, H., Valdivia, M., Palacios, J., \& Romero, H. (2018). Metodología de la Investigación. Bogotá - México: Ediciones de la U.

Pérez Jaramillo, C. M. (s.f.). Indicadores de Gestión. Obtenido de Soporte \& CIA. LTDA: https://www.visionadministrativa.info/biblioteca/control/indicadores/losindicado resdegestion.pdf

Ponce Talancón, H. (2007). La matriz foda: alternativa de diagnóstico y determinación de estrategias de intervención en diversas. Enseñanza e Investigación en Psicología, 19.

Presidencia Constitucional Interina de la República del Ecuador. (2004). Reglamento General de la Ley de Defensa del Artesano. Quito.

Robbins, S., \& Coulter, M. (2005). Administración. Octava edición. Mexico: PEARSON EDUCACIÓN.

Ronda Pupo, G. A. (11 de marzo de 2021). Estrategia. Qué es, origen, definición según autores, tipos. Obtenido de Gestiopolis: https://www.gestiopolis.com/unconcepto-de-estrategia/

Sánchez Galán, J. (29 de septiembre de 2020). Funciones Administrativas. Obtenido de Economipedia: https://economipedia.com/definiciones/funcionesadministrativas.html

Sarli, R. R., González, S. I., \& Ayres, N. (2015). Análisis FODA. Una herramienta necesaria. Obtenido de Universidad Nacional de Cuyo: https://tesisenfermeria.bdigital.uncu.edu.ar/objetos_digitales/7320/sarlirfo912015.pdf

Saroka, R., \& Ferrari, C. (Ediciones Macchi). LOS ORGANIGRAMAS, DISEÑO E INTERPRETACIÓN. Buenos Aires: 2015.

Thompson, A. A., Gamble, J. E., Peteraf, M. A., \& Strickland III, A. J. (2012). Administración Estratégica: Teoría y casos. México: McGraw-Hill Interamericana.

Villagra Villanueva, J. A. (2016). Indicadores de Gestión. Un enfoque práctico. Santa Fe: Cengage Learning Editores. 